\title{
Product Demand Forecasting and Dynamic Pricing considering Consumers' Mental Accounting and Peak-End Reference Effects
}

\author{
Wenjie $\mathrm{Bi}^{1}$ and Mengqi Liu ${ }^{2}$ \\ ${ }^{1}$ Business School, Central South University, Changsha 410083, China \\ ${ }^{2}$ Business School, Hunan University, Changsha 410082, China \\ Correspondence should be addressed to Mengqi Liu; liumengqi1976@163.com
}

Received 19 May 2014; Accepted 17 July 2014; Published 2 September 2014

Academic Editor: Li Guo

Copyright (c) 2014 W. Bi and M. Liu. This is an open access article distributed under the Creative Commons Attribution License, which permits unrestricted use, distribution, and reproduction in any medium, provided the original work is properly cited.

\begin{abstract}
We introduce a demand forecasting model for a monopolistic company selling products to consumers with double-entry mental accounting, which means consumers experience pleasure when consuming goods or service and feel pains when paying for them. Moreover, as the monopolist changes prices, consumers form a reference price that adjusts an anchoring standard based on the lowest price that they perceived, namely, the peak-end anchoring. We obtain the steady state prices under three different payment schemes for two- and infinite-period. We also analyze the relationship between these steady prices and maximal profit and compare the steady state prices of different payment schemes by changing the double-entry mental accounting's parameters through numerical examples. The proposed model is computationally tractable for demand forecasting of realistic size.
\end{abstract}

\section{Introduction}

Accurate demand forecasts are essential for companies including manufactures and distributors because they will drive more responsive customer service with lower inventories and reduced obsolescence $[1,2]$. As Huang et al. [3] have shown, price-dependent demand models are the most commonly employed possibly because pricing strategy is the most effective tool that has been used to impact a firm's demand. Under consideration of an integrated demand and supply chain management, forecasting and pricing are related to each other. Therefore, we obviously should consider the impact of its price when predicting the consumers' demand of a certain product. A distinctive feature of this work is that it depends on descriptive models of consumer behavior to predict customers' demand and to derive pricing strategies under dynamic settings. Specifically, we incorporate both the consumers' mental accounting and the impact of reference price when modeling our demand function.

Traditional assumption of consumers' rationality sometimes cannot explain the realistic, complex world $[4,5]$. In order to make accurate prediction about product purchase, we try to study the consumers' behavior impact on it. Mental accounting is an important concept in behavioral economics, which is the set of cognitive operations used by individuals and households to organize, evaluate, and keep track of financial activities [6]. And mental accounting theory can explain many economic anomalies about price such as the endowment effect and the sunk cost effect [7]. Since mental accounting was first formally proposed by Thaler in 1985 [8], it has been widely studied. One of the most outstanding models is the double-entry mental accounting model proposed by Prelec and Loewenstein [9], which obtain a variety of predictions that are against the traditional economics theory such as debt aversion and preferences for prepayment. The double-entry model describes the nature of the reciprocal interactions between the pleasure of consumption and the pain of paying and introduces the idea of prospective accounting and coupling which refers to the degree to which consumption calls to mind thoughts of payment and vice versa. Besides, the double-entry model introduces two coupling coefficients, that is, pleasure attenuation coefficient $\psi$ and pain buffering coefficient $\gamma$, which represent and respect the degree to which payments attenuate the pleasure of consumption and the degree to which consumption buffers the pain of payments, respectively. And there are other important studies on mental accounting; see [10-16]. All these studies belong to empirical research, so it is essentially 
needed to explore how to incorporate mental accounting into theoretical models in economics, and now there have been a small number of behavioral operations management studies attempting to model mental accounting. Erat and Bhaskaran [17] formulate a simple model to formalize how (and why) the mental account associated with a base product impacts a consumers' add-on purchase decision, and they also develop a normative model to explicitly examine what (if any) implications the proposed consumer biases have on firm's pricing decisions. And Chen et al. [18] studied what effects the payment schemes have on inventory decisions in the newsvendor problem when considering mental accounting and found out that "prospective accounting" in the double-entry mental accounting model can explain that when keeping the net profit structure constant, inventory quantities exhibit a consistent decreasing pattern in the order of payment schemes $\mathrm{O}$ (where the order is financed by the newsvendor herself), $\mathrm{S}$ (where the order is financed by the supplier through delayed order payment), and C (where the order is financed by the customer through advanced revenue). To sum up, mental accounting is closely related to consumer behavior, but current studies on mental accounting are mostly empirical research, and there are seldom dynamic pricing models incorporating double-entry mental accounting yet.

On the other hand, mental accounting is always closely linked with reference price. According to [19], consumers keep a reference price in mind and perform two comparisons. First, they compare the reference price with the actual price and this comparison yields transaction utility. Second, they compare the benefits of consumption with the reference price yielding acquisition utility. And it is essential to note that the demand model in [20] is exactly established on this framework. Moreover, Baucells and Hwang [7] propose the MARA model of multiperiod purchase decision-making, which integrates the psychological mechanism of mental accounting and reference price adaptation. And the MARA model can capture some important underlying psychological processes (such as payment depreciation) that other mental accounting models (including Thaler's double-comparison model and Prelec and Loewenstein's double-entry mental accounting model) fail to do. Therefore, it is necessary to simultaneously incorporate consumers' mental accounting and reference-dependent behavior into dynamic pricing models. In particular, under the influence of double-entry mental accounting, not only the reference price will be constantly updated, but also the consumers' perceived price and perceived consumption benefit will be changed under different payment schemes; consequently, the demand function will vary, thus affecting the prediction of product demand quantity. This suggests that there may exist further research opportunities for using the combination of consumers' double-entry mental accounting and reference effect to forecast the demand of a product and to investigate the optimal pricing strategy of a monopolistic firm.

The remainder of this paper is organized as follows. Section 2 describes our model of double-entry mental accounting in product demand forecasting and further investigates the combined effect of consumers' double-entry mental accounting and reference-dependent behavior on firm's pricing strategies. Section 3 provides two-period dynamic pricing model under three different payment schemes and drives an explicit solution. In Section 4, we study infinite-period dynamic pricing model. Section 5 reports the numerical study and Section 6 concludes the paper with a summary of results.

\section{The Basic Model}

Consider a product sold by a monopolistic company over an infinite horizon through three different payment schemes. The first payment scheme (scheme $O$ ), the most ordinary one, indicates that consumers pay for and consume a product simultaneously in each period. The second one is prepayment (scheme Pre) meaning that consumers pay at the beginning and consume at the end of each period, and the third scheme postpayment (scheme Post) shows completely opposite conditions of prepayment that consumers consume at first and pay in the end. And the impact of doubleentry mental accounting on consumer is different under different payment schemes. In this section, we build demand forecasting and dynamic pricing models under each payment scheme and compare their impacts on the monopolist's profit.

To facilitate the analysis, we assume that product demand function is linear, as shown in Assumption 1.

Assumption 1. The reference-dependent demand is $D(p, r)=$ $q(p)+R(r-p, r)$ where $q(p)=\beta_{0}-\beta_{1} p, R(r-p, r)=$ $\beta_{2} \min (r-p, 0)+\beta_{3} \max (r-p, 0)$, and $\beta_{0}, \beta_{1}, \beta_{2}, \beta_{3} \geq 0$. Let the price interval be $P=[p, \bar{p}]$ and let the product cost be 0 .

The term $\beta_{0}, \beta_{1}, \beta_{2}, \beta_{3} \geq 0$ ensures that the demand function is decreasing in price and increasing in reference price. For loss-averse consumers, the demand function is steeper for losses than for gains; for example, $\beta_{2}>\beta_{3}$ while $\beta_{2}<\beta_{3}$ for loss-seeking consumers. And for loss-neutral consumers, we have $\beta_{2}=\beta_{3}$ so the demand function is smooth.

According to [20], $R(x, r)$ measures the impact on demand of a perceived discount/surcharge where $x=r-p$, relative to the reference price $r$. And it can be seen from Assumption 1 that $R(x, r) \geq 0$ for $x>0, R(x, r) \leq 0$ for $x<0$, and $R(0, r)=0$.

Let $\Pi(p, r)=p q(p)+p R(r-p, r)$ be the short-term profit where $\pi_{0}(p)=p q(p)$ is the base profit without reference effect and $\Pi^{R}(p, r)=p R(r-p, r)$ is the profit from the reference effect. Let $\kappa(r)=R_{x}(0, r)$ denote the slope of the reference demand at $x=0$ when consumers are loss neutral. Next, we give a typical technical assumption on $\Pi(p, r)$ borrowed from Assumption 3 in [20].

Assumption 2. (a) $\pi(p)$ is nonmonotonic and concave in $p$. (b) $\prod_{p}(r, r)=\pi^{\prime}(r)-r \kappa(r)$ is strictly decreasing in $r$. (c) $\Pi^{R}(p, r)$ is concave in $p$ and supermodular in $(p, r)$. 
The reference price formation and updating mechanism in this paper is assumed to be peak-end anchoring, as shown in Assumption 3, which is the most commonly used and empirically validated reference price mechanism in the literature; for example, see [21,22].

Assumption 3. The reference price updating mechanism is given by $r_{t}=\eta m_{t-1}+(1-\eta) p_{t-1}$ where $m_{t-1}=$ $\min \left(m_{0}, p_{1}, \ldots, p_{t}\right)=\min \left(m_{t-2}, p_{t-1}\right), t>1, r_{1}=m_{0}$, and the memory parameter $\eta \in[0,1]$ captures the fraction of consumers anchoring on the lowest price.

Based on this assumption we can know that for $\eta=0$ the model becomes a special case where the consumers anchor solely on the previous period price.

Given initial conditions $m_{0}$ and $p_{0}$ (we can regard $p_{0}$ as $m_{0}$ ), the monopolist maximizes infinite horizon $\beta$ discounted revenues

$$
\begin{gathered}
v\left(m_{0}, p_{0}\right)=\max _{p \in P} \sum_{t=1}^{\infty} \beta^{t} \prod\left(p_{t}, r_{t}\right), \\
r_{t}=\eta m_{t-1}+(1-\eta) p_{t-1}, \quad m_{t}=\min \left(m_{t-1}, p_{t}\right),
\end{gathered}
$$

where $\beta \in[0,1]$ is the firm's discount factor.

The Bellman equation for this problem is

$$
\begin{aligned}
v\left(m_{t-1}, p_{t-1}\right)=\max _{p \in P} \sum_{t=1}^{\infty}\{ & \prod\left(p_{t}, r_{t}\right) \\
& \left.+\beta V\left(\min \left(p_{t}, m_{t-1}\right), p_{t}\right)\right\} .
\end{aligned}
$$

We can know from Lemma 1 of [23] that the value function $V(m, p)$ is increasing in both arguments.

The infinite horizon model implicitly assumes that lowest prices can be remembered indefinitely. This is a reasonable approximation in a context where the frequency of transactions is high relative to the horizon length and the lowest prices are recalled because of their salience; their extremeness makes them stand out in the memory process.

Next, we analyze how different payment schemes influence consumers' perceived price and perceived consumption benefit. Table 1 describes, under prepayment scheme and postpayment scheme, how consumers' perceived price $\widehat{p}$ and perceived consumption benefit $\hat{\theta}$ are influenced by pleasure attenuation coefficient $\psi$, pain buffering coefficient $\gamma$, and consumers' discount factor $\varphi$ for product price and consumption benefit because of the separation of consumption and payment where $\psi, \gamma$, and $\varphi \in[0,1]$.

On the basis of Prelec and Loewenstein's [9] doubleentry mental accounting model, we can account for Table 1 as follows.

(1) Under prepayment scheme, consumers pay price $p$ at first and their prospective accounting will think of future consumption benefits so that the pain of payments will be buffered, which leads perceived payment to be $(1-\gamma) p$. On the other hand, because of the delay of consumption, the perceived consumption benefit will be at discount and will become $\varphi \theta$.
TABLE 1: Perceived price $\widehat{p}$ and perceived consumption benefit $\widehat{\theta}$.

\begin{tabular}{lcc}
\hline & Prepayment & Postpayment \\
\hline Consumption & $\widehat{\theta}=\varphi \theta$ & $\widehat{\theta}=(1-\psi) \theta$ \\
Payment & $\widehat{p}=(1-\gamma) p$ & $\widehat{p}=\varphi p$ \\
\hline
\end{tabular}

(2) Under postpayment scheme, consumers obtain consumption benefit $\theta$ at first and the prospective accounting will consider future payments so that the pleasure of consumption today will be attenuated, which leads perceived consumption benefit to be $(1-\psi) \theta$. On the other hand, because of the delay of payment, the perceived price will be at discount and will become $\varphi p$.

Under the influence of mental accounting, consumers will make decision depending on perceived price and perceived consumption benefit instead of actual price and consumption benefit. Then we can know that when considering the dynamic pricing problem with consumers' double-entry mental accounting and reference-dependent behavior, the demand function $D_{\text {pre }}(p, r)$ and $D_{\text {post }}(p, r)$ can be expressed as follows:

$$
\begin{gathered}
D_{\text {pre }}(p, r)=D((1-\gamma) p, r), \\
D_{\text {pre }}(p, r)=D(\varphi p, r) .
\end{gathered}
$$

And accordingly, the updating of reference price is affected by perceived price instead of actual price; that is, $r_{t}=\eta m_{t-1}+(1-\eta) p_{t-1}, m_{t-1}=\min \left(m_{t-2}, p_{t-1}\right)$.

Based on the above assumptions, the monopolist's profit $\Pi_{\text {pre }}(p, r)$ under prepayment scheme is

$$
\begin{aligned}
\prod_{\text {pre }}(p, r) & =p D_{\text {pre }}(p, r) \\
& =\frac{1}{1-\gamma} \pi(r-(1-\gamma) p)+p R(r-(1-\gamma) p, r)
\end{aligned}
$$

so that (2) under prepayment scheme can be rewritten as

$$
V_{\text {pre }}(r, p)=\max _{p \in P} \prod_{\text {pre }}(p, r)+\beta V_{\text {pre }}(\eta r+(1-\eta) \hat{p}) .
$$

Similarly, the monopolist's profit $\Pi_{\text {post }}(p, r)$ under postpayment scheme is

$$
\prod_{\text {post }}(p, r)=p D_{\text {post }}(p, r)=\frac{1}{\varphi} \pi(\varphi p)+p R(r-\varphi p, r)
$$

so that (2) under postpayment scheme can be rewritten as

$$
V_{\text {post }}(r, p)=\max _{p \in P} \prod_{\text {post }}(p, r)+\beta V_{\text {post }}(\eta r+(1-\eta) \hat{p}) .
$$

We assume consumers are loss neutral; namely, $\beta_{2}=\beta_{3}$. Then according to Assumption 1 we have

$$
\begin{gathered}
D_{\text {pre }}(p, r)=\beta_{0}-\beta_{1}(1-\gamma) p+\beta_{2}(r-(1-\gamma) p), \\
D_{\text {post }}(p, r)=\beta_{0}-\beta_{1} \varphi p+\beta_{2}(r-\varphi p) .
\end{gathered}
$$




\section{The Two-Period Dynamic Pricing Model}

The two-period dynamic pricing model is the simplest and commonly used in practice. Generally, it is easy to calculate the analytical solution of optimal price path. Also the obtained properties and conclusions are clear at a glance and good for interpretation. Hence, we start with studying the two-period model.

Based on the analysis in Section 2, the single-period profits under scheme $O$, scheme Pre (prepayment scheme), and scheme Post (postpayment scheme) in two-period model $\Pi_{o}\left(p_{t}, r_{t}\right), \Pi_{\text {pre }}\left(p_{t}, r_{t}\right)$, and $\Pi_{\text {post }}\left(p_{t}, r_{t}\right)$ can expressed below $(t=1,2)$, respectively:

$$
\begin{gathered}
\prod_{0}\left(p_{t}, r_{t}\right)=p_{t}\left[\beta_{0}-\beta_{1} p_{t}+\beta_{2}\left(r_{t}-p_{t}\right)\right] \\
\prod_{\text {pre }}\left(p_{t}, r_{t}\right)=p_{t}\left[\beta_{0}-\beta_{1}(1-\gamma) p_{t}+\beta_{2}\left(r_{t}-(1-\gamma) p_{t}\right)\right]
\end{gathered}
$$

$$
\prod_{\text {post }}\left(p_{t}, r_{t}\right)=p_{t}\left[\beta_{0}-\beta_{1} \varphi p_{t}+\beta_{2}\left(r_{t}-\varphi p_{t}\right)\right]
$$

As a result, the two-period dynamic pricing models under scheme $O$, scheme Pre, and scheme Post are $(t=1,2)$

$$
\begin{gathered}
V_{0}\left(m_{0}\right)=\sup _{p \in P} \prod_{0}\left(p_{1}, r_{1}\right)+\beta V_{0}\left(p_{2}, r_{2}\right), \\
r_{2}=\eta m_{1}+(1-\eta) p_{1}, \quad m_{1}=\min \left(m_{0}, p_{1}\right), \\
V_{\text {pre }}\left(m_{0}\right)=\sup _{p \in P} \prod_{\text {pre }}\left(p_{1}, r_{1}\right)+\beta V_{\text {pre }}\left(p_{2}, r_{2}\right), \\
r_{2}=\eta m_{1}+(1-\eta)(1-\gamma) p_{1}, \quad m_{1}=\min \left(m_{0},(1-\gamma) p_{1}\right), \\
V_{\text {post }}\left(m_{0}\right)=\sup _{p \in P} \prod_{\text {post }}\left(p_{1}, r_{1}\right)+\beta V_{\text {post }}\left(p_{2}, r_{2}\right), \\
r_{2}=\eta m_{1}+(1-\eta) \varphi p_{1}, \quad m_{1}=\min \left(m_{0}, \varphi p_{1}\right) .
\end{gathered}
$$

Proposition 4. Given the initial reference price $m_{0}$, let $\left\{p_{o, 1}^{*}, p_{o, 2}^{*}\right\},\left\{p_{p r e, 1}^{*}, p_{\text {pre }, 2}^{*}\right\}$, and $\left\{p_{\text {post }, 1}^{*}, p_{\text {post }, 2}^{*}\right\}$ be the optimal price path of models (12), (13), and (14), respectively.

Case 1. If $m_{0}$ is large enough satisfying $m_{0}=\max \left(m_{0}, p,(1-\right.$ $\left.\gamma) p_{1}, \varphi p_{1}\right)$, we obtain

$$
\begin{aligned}
& p_{\text {pre }, 1}^{*}=\frac{p_{0.1}^{*}}{1-\gamma}, \quad p_{\text {pre }, 2}^{*}=\frac{p_{0.2}^{*}}{1-\gamma} p_{\text {post }, 1}^{*}=\frac{p_{0.1}^{*}}{\varphi}, \\
& p_{\text {post }, 2}^{*}=\frac{p_{0.2}^{*}}{\varphi},
\end{aligned}
$$

where

$$
\begin{aligned}
& p_{0,1}^{*}=\frac{2\left(\beta_{0}+\beta_{2} m_{0}\right)\left(\beta_{1}+\beta_{2}\right)+\beta \beta_{0} \beta_{2}}{4\left(\beta_{1}+\beta_{2}\right)^{2}-\beta \beta_{2}^{2}}, \\
& p_{0,2}^{*}=\frac{\beta_{0}+\beta_{2} p_{0.1}^{*}}{2\left(\beta_{1}+\beta_{2}\right)} .
\end{aligned}
$$

Case 2. If $m_{0}$ is small satisfying $m_{0}=\min \left(m_{0}, p_{1},(1-\right.$ $\left.\gamma) p_{1}, \delta p_{1}\right)$, we also have

$$
\begin{aligned}
& p_{\mathrm{pre}, 1}^{*}=\frac{p_{0.1}^{*}}{1-\gamma}, \quad p_{\mathrm{pre}, 2}^{*}=\frac{p_{0.2}^{*}}{1-\gamma} p_{\mathrm{post}, 1}^{*}=\frac{p_{0.1}^{*}}{\varphi}, \\
& p_{\mathrm{post}, 2}^{*}=\frac{p_{0.2}^{*}}{\varphi},
\end{aligned}
$$

where

$$
\begin{gathered}
p_{0,1}^{*}=\frac{2\left(\beta_{0}+\beta_{2} m_{0}\right)\left(\beta_{1}+\beta_{2}\right)+\beta\left(\beta_{0}+\beta_{2} \eta m_{0}\right) \beta_{2}(1-\eta)}{4\left(\beta_{1}+\beta_{2}\right)^{2}-\beta \beta_{2}^{2}(1-\eta)^{2}}, \\
p_{0,2}^{*}=\frac{\beta_{0}+\beta_{2}\left(\eta m_{0}+(1-\eta) p_{0.1}^{*}\right)}{2(1-\beta)\left(\beta_{1}+\beta_{2}\right)} .
\end{gathered}
$$

Proof. See Appendices A, B, and C.

By Proposition 4, the ratios of each period's optimal price to the corresponding period's optimal price under scheme $O$ are identical. In other words, if the optimal price path under scheme $O$ is $\left\{p_{o, 1}^{*}, p_{o, 2}^{*}\right\}$, the corresponding optimal paths of scheme Pre will be $\left\{p_{o, 1}^{*} /(1-\gamma), p_{o, 2}^{*} /(1-\gamma)\right\}$ and the corresponding optimal paths of scheme Post will be $\left\{p_{o, 1}^{*} / \varphi, p_{o, 2}^{*} / \varphi\right\}$.

After obtaining the optimal price paths, we can compare firm's profits under different payment schemes and provide theoretical support for firm's decision on how to choose payment scheme for higher profit.

Proposition 5. Given the initial reference price $m_{0}$, let $V_{0}^{*}\left(m_{0}\right), V_{\text {pre }}^{*}\left(m_{0}\right)$, and $V_{\text {post }}^{*}\left(m_{0}\right)$ be the maximal profit of models (12), (13), and (14), respectively. They satisfy the following equations:

$$
V_{\text {pre }}^{*}\left(m_{0}\right)=\frac{V_{0}^{*}\left(m_{0}\right)}{1-\gamma}, \quad V_{\text {post }}^{*}\left(m_{0}\right)=\frac{V_{0}^{*}\left(m_{0}\right)}{\varphi} .
$$

Proof. See Appendices A, B, and C.

Based on Proposition 5 it is straightforward to draw the following conclusions.

(1) If $\varphi>1-\gamma$, it will be better for the monopolist to provide prepayment scheme to consumers, or postpayment scheme.

For scheme $O$, the pleasure of consumption and the pain of paying are equal. And under scheme Pre, the bigger $\gamma$ is, the more buffered the pain of paying is because of mental accounting; and the bigger $1-\varphi$ is, the more attenuated the pleasure of consumption is because of time discounting. As a result, $\varphi>1-\gamma$ means the reduced pain is less than the reduced pleasure, and the benefit outweighs the disadvantage, which leads to the increasing in demand. And hence, it is profitable for providing scheme Pre where the firm's profit becomes higher. Similarly, we can explain the attractiveness of scheme Post in the case of $\varphi \leq 1-\gamma$. 
(2) For $\varphi=1-\gamma$, scheme Pre and scheme Post are indifferent in pricing strategies and profitability.

When $\varphi=1-\gamma$, the effects of pain buffering coefficient $\gamma$ and consumers' discount factor $\varphi$ are offset reciprocally. Consequently, we have $V_{\text {pre }}^{*}\left(m_{0}\right)=V_{\text {post }}^{*}\left(m_{0}\right)$.

(3) In general, $\gamma, \varphi \in[0,1]$; namely, $V_{\text {pre }}^{*}\left(m_{0}\right)>V_{0}^{*}\left(m_{0}\right)$ and $V_{\text {post }}^{*}\left(m_{0}\right)>V_{0}^{*}\left(m_{0}\right)$, so it is usually better to choose the scheme Pre and scheme Post.

\section{The Infinite-Period Dynamic Pricing Model}

With the fierce change of the market environment and the rapid development of Internet, price adjustments become more and more frequent and periodicity of pricing is growing fast. Hence, the pricing strategies and their related properties of the multiperiod dynamic pricing problem and the infiniteperiod dynamic pricing problem are worthy of further study. In this section, we study the infinite-period dynamic pricing problem considering consumers' double-entry mental accounting and reference-dependent behavior.

4.1. The Steady State. This section characterizes the long-term pricing strategy of the firm facing loss-neutralconsumers with demand given by Assumption 1 where $\beta_{2}=\beta_{3}$.

First, we will consider the scheme $O$ in which case the Bellman equation is given in (2). To get the steady states of (2) requires a nonstandard approach, because the transition in the value function (memory structure) is nonsmooth. Our analysis is based on a bounding technique [23], which identifies the steady states of (2) based on those of a series of smooth problem.

For $w \in[0,1]$ and $m \in P$ consider the following smooth problem with one-dimensional state:

$$
\begin{gathered}
V_{m}^{v}\left(p_{t-1}\right)=\max _{p_{t} \in P}(1-w) \prod\left(p_{t}, \eta m_{t-1}+(1-\eta) p_{t-1}\right) \\
+w \prod\left(p_{t}, p_{t-1}\right)+\beta V_{m}^{v}\left(p_{t}\right) .
\end{gathered}
$$

We first show that the family $V_{m}^{w}, w \in[0,1]$, provides upper bounds for the value function $V$. Based on [23], we can know that for any $m<p$, we have $V(m, p) \leq V_{m}^{w}(p)$.

We next argue that by approximating the value function $V$ by a smooth upper bound $V_{m}^{w}$, for an appropriate subset of value $V$, the firm will charge optimal prices in the long run. Technically, this amounts to matching supergradients of the original problem with gradients for an appropriate smooth upper bound equation (2). We first identify steady states of problem (20) which will help characterize those of (2).

The structure of the problem leads us to consider three price-memory scenarios (low, medium, and high): $R_{1}=[p, s], R_{2}=[s, S]$, and $R_{3}=[s, S]$ where the thresholds $s, S$ solve, respectively,

$$
\begin{gathered}
\pi_{0}^{\prime}(p)-\beta_{2}(1-\beta(1-\eta) p)=0, \\
\pi_{0}^{\prime}(p)-\beta_{2}(1-\beta p)=0,
\end{gathered}
$$

where $\pi_{0}(p)=p q(p)$ represents the base profit

Uniqueness of $s$ and $S$ follows because the above left hand sides (LHS) are strictly decreasing in $p$, by concavity of $\pi_{0}^{\prime}(p)$. To understand $s, S$ better, we will give Lemma 6 in which (21) and (22) are special case.

Lemma 6. (a) For $w \in[0,1]$ and $m \in P$, (2) under scheme $O$ admits a unique steady state, which solves

$$
\begin{aligned}
\pi_{0}^{\prime}(p) & -\beta_{2}\left[(1-w)\left(2-(1-\eta)(1+\beta)+\beta_{2} w(1-\beta)\right)\right] p \\
+ & \beta_{2}(1-w) \eta m=0 .
\end{aligned}
$$

(b) For any $m \in[s, S]$, there exists $w \in[0,1]$ such that $m$ is a steady state of the corresponding equation (20).

Proof. See Lemma 4 of [23].

Denote $p^{* *}(m)$ the unique steady state of problem (20) for $w=0$ by Lemma $6(\mathrm{a}) . p^{* *}(m)$ solves

$$
\pi_{0}^{\prime}(p)-\beta_{2}(2-(1-\eta)(1+\beta)) p+\beta_{2} \eta m=0 .
$$

In particular $p^{* *}(s)=s$; the thresholds $s$ and $S$ defined above correspond to those values $m$ for which the steady state of $J_{m}^{w}$ equals $m$, for $w=0$, respectively, $w=1$. It turns out that $(s, s)$ and $(S, S)$ are steady state of our equation (2). The next result identifies steady states of (2) based on the steady states of (20), identified in Lemma 6.

Based on Lemma 6, we can get Proposition 7 which gives the steady state.

Proposition 7. (a) For $m \in R_{1},\left(m, p^{* *}(m)\right)$ is a steady state of (2) where $p^{* *}(m)$ solves $(10)$. (b) For $m \in R_{2},(m, m)$ is a steady state of (6).

Proof. See Appendices A, B, and C.

Proposition 7 suggests to partition the initial states space into the following region: $\overline{R_{1 a}}=\left\{(m, p) \mid p \geq p^{* *}(m), m \leq\right.$ $s\}, \overline{R_{1 b}}=\left\{(m, p) \mid p \leq p^{* *}(m), m \leq s\right\}, \overline{R_{2}}=\{(m, p) \mid p \geq$ $\left.p^{* *}(m), s \leq m \leq S\right\}$, and $\overline{R_{3}}=\{(m, p) \mid p \geq m, m \geq S\}$.

The main results in Proposition 7 are indeed the only steady state of (2).

The result says that the lower the value of the steady state is, the more sensitive consumers are to deviations from the reference price. Furthermore, a more patient firm (higher $\beta$ ) charges higher steady state prices.

Based on the analysis above, we can give the similar conclusions on the scheme Pre and scheme Post. Under the scheme Pre let us consider the following smooth problem with one-dimensional state which is similar to (5):

$$
\begin{aligned}
V_{\text {pre.m }}^{v}\left(p_{t-1}\right)= & \max _{p_{t} \in P}(1-\omega) \prod_{\text {pre }}\left(p_{t}, \eta m+(1-\eta) p_{t-1}\right) \\
& +\omega \prod_{\text {pre }}\left(p_{t}, p_{t-1}\right)+\beta V_{\text {pre. } m}^{v}\left(p_{t}\right) .
\end{aligned}
$$


Similarly, we can obtain that $V_{\text {pre }} V(m, p) \leq V_{\text {pre.m }}^{\omega}(p)$. Now we get Proposition 8 which gives the steady state of (5). The proof is similar to Proposition 7 and is omitted here.

Proposition 8. (a) For $w \in[0,1]$ and $p \in P$, (5) under scheme Pre admits a unique steady state, which solves the following equations:

$$
\begin{aligned}
& \frac{1}{1-\gamma} \pi_{0}^{\prime}((1-\gamma) p) \\
& \quad-\beta_{2}(1-\gamma)[(1-\omega)((2-(1-\eta)(1+\beta))+\omega(1-\beta))] p \\
& \quad+\beta_{2}(1-\omega) \eta m=0 .
\end{aligned}
$$

(b) For any $m \in\left[s_{\text {pre }}, S_{\text {pre }}\right]$, there exists $w \in[0,1]$ such that $m$ is a steady state of the corresponding equation (25), and $s_{\text {pre }}, S_{\text {pre }}$ solve the following equations, respectively:

$$
\begin{aligned}
& \frac{1}{1-\gamma} \pi_{0}^{\prime}((1-\gamma) p) \\
& -\beta_{2}(1-\gamma)(2-(1-\eta)(1+\beta)) p \\
& \quad+\beta_{2} \eta p=0 ; \\
& \frac{1}{1-\gamma} \pi_{0}^{\prime}((1-\gamma) p)-\beta_{2}(1-\gamma)(1-\beta) p=0 .
\end{aligned}
$$

Also denote by $p_{\text {pre }}^{* *}(m)$ the unique steady state of problem (25) for $w=0$. By Lemma 6(a), $p^{* *}(m)$ solves

$$
\begin{aligned}
& \frac{1}{1-\gamma} \pi_{0}^{\prime}((1-\gamma) p) \\
& \quad-\beta_{2}(1-\gamma)(2-(1-\eta)(1+\beta)) p \\
& \quad+\beta_{2} \eta m=0 .
\end{aligned}
$$

Similarly, we can get that $\left(s_{\text {pre }}, s_{\text {pre }}\right)$ and $\left(S_{\text {pre }}, S_{\text {pre }}\right)$ are steady state of our equation (5).

For the scheme Post, we also use the one-dimensional state which is similar to (7) as follows:

$$
\begin{aligned}
V_{\text {post. } m}^{v}\left(p_{t-1}\right)= & \max _{p_{t} \in P}(1-\omega) \prod_{\text {post }}\left(p_{t}, \eta m+(1-\eta) p_{t-1}\right) \\
& +\omega \prod_{\text {post }}\left(p_{t}, p_{t-1}\right)+\beta V_{\text {post. } m}^{v}\left(p_{t}\right)
\end{aligned}
$$

Similarly, we can obtain that $V_{\text {post }} V(m, p) \leq V_{\text {post. } m}^{\omega}(p)$. Now we obtain Proposition 9 which gives the steady state of (7). The proof is also similar to Proposition 7 and is omitted here.

Proposition 9. (a) For $w \in[0,1]$ and $p \in P$, (7) under scheme Post admits a unique steady state, which solves the following equations:

$$
\begin{aligned}
\frac{1}{\delta} \pi_{0}^{\prime}(\delta p)-\beta_{2} \delta[ & (1-\omega)(2-(1-\eta)(1+\beta)) \\
& +\omega(1-\beta)] p+\beta_{2}(1-\omega) \eta m=0 .
\end{aligned}
$$

(b) For any $m \in\left[s_{\text {post }}, S_{\text {post }}\right]$, there exists $w \in[0,1]$ such that $m$ is a steady state of the corresponding equation (29), and $s_{\text {post }}, S_{\text {post }}$ solve the following equations, respectively:

$$
\begin{gathered}
\frac{1}{\delta} \pi_{0}^{\prime}(\delta p)-\beta_{2} \delta(2-(1-\eta)(1+\beta)) p+\beta_{2} \eta p=0 \\
\frac{1}{\delta} \pi_{0}^{\prime}(\delta p)-\beta_{2} \delta(1-\beta) p=0
\end{gathered}
$$

We denote $p_{\text {post }}^{* *}(m)$ as the unique steady state of problem (29) for $w \in[0,1]$. By Lemma 6(a), $p_{\text {post }}^{* *}(m)$ solves

$$
\frac{1}{\delta} \pi_{0}^{\prime}(\delta p)-\beta_{2} \delta(2-(1-\eta)(1+\beta)) p+\beta_{2} \eta m=0 .
$$

4.2. The Optimal Policy and Price Paths. This section investigates the transient pricing policy of the monopolist. Firstly, we study convergence and monotonicity of the price paths of (2); then we can use the similar way to analyze (5) and (7), namely, under the scheme Pre and scheme Post. We start at an arbitrary initial state $\left(m_{0}, p_{0}\right)$, in which $m_{0} \leq p_{0}$ and $m_{0}$ can be regarded as $p_{0}$. The optimal pricing policy of the monopolist is

$$
\begin{aligned}
p^{*}\left(m_{t-1}, p_{t-1}\right)= & \underset{p_{t} \in P}{\arg \max } \prod\left(p_{t}, \eta m+(1-\eta) p_{t-1}\right) \\
& +\beta V\left(\min \left(m_{t-1}, p_{t}\right), p_{t}\right) .
\end{aligned}
$$

The optimal price path $\left\{p_{t}\right\}_{t}$ is given by $p_{t}=p^{*}\left(m_{t-1}, p_{t-1}\right)$ with $m_{t}=\min \left(m_{t-1}, p_{t}\right), t \geq 1$, and the state path is $\left\{\left(m_{t}, p_{t}\right)\right\}$.

Our first result in this section shows that if $\left(m_{0}, p_{0}\right)$ is in any of the three regions $R_{t}, t=1,2,3$, as defined in Section 4.1, the state path remains in that region. We can know that [23] if $m_{0} \in R_{1} \cup R_{2}$, then $p_{t} \geq m_{0}$ for all $t$. If $m_{0} \in$ $R_{3}$, then $m_{t} \in R_{3}$ for all $t$. Under the scheme Pre and scheme Post, we can get the similar conclusions as Proposition 10 and the proof is similar to Proposition 7.

Proposition 10. (a) For the scheme Pre, if $m_{0} \in R_{\text {pre. } 1} \cup R_{\text {pre. } 2}$, then $p_{t} \geq m_{0}$ for all $t$. If $m_{0} \in R_{\text {pre. } 3}$, then $m_{t} \in R_{\text {pre. } 3}$ for all $t$, where $R_{\text {pre. } 1}=\left[\underline{p}, s_{\text {pre }}\right], R_{\text {pre. } 2}=\left[s_{\text {pre }}, S_{\text {pre }}\right], R_{\text {pre. } 3}=\left[s_{\text {pre }}, \bar{p}\right]$, and $s_{\text {pre }}, S_{\text {pre }}$ are defined in Section 4.1.

(b) For the scheme Post, if $m_{0} \in R_{\text {post. } 1} \cup R_{\text {post. } 2}$, then $p_{t} \geq$

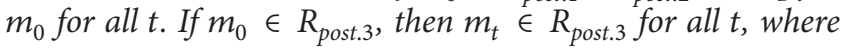
$R_{\text {post. } 1}=\left[\underline{p}, s_{\text {post }}\right], R_{\text {post. } 3}=\left[s_{\text {post }}, \bar{p}\right]$, and $s_{\text {post }}, S_{\text {post }}$ are defined in Section 4.1 . 
The first part of these three propositions shows that if the initial minimum price $m_{0}$ is not too high, the optimal price path stays above $m_{0}$ : for the scheme $O$, if $m_{0}<S$ (for the scheme Pre, if $m_{0}<S_{\text {pre }}$; for the scheme Post, if $m_{0}<S_{\text {post }}$ ) the minimal price does not change over time, and so the state path will remain within the region. On the other hand if the initial minimal price $m_{0}$ is relatively high, for the scheme $O$, if $m_{0}>S$ (for the scheme Pre, if $m_{0}>S_{\text {pre }}$; for the scheme Post, if $m_{0}>S_{\text {post }}$ ), the minimum price decreases over time, but it never drops below $S$ under scheme $O, S_{\text {pre }}$ under scheme Pre, and $S_{\text {post }}$ under scheme Post. These results identify the possible convergence points of the optimal price paths, starting at any initial state.

Proposition 10 implies that if the optimal price path of (2) under scheme $O$ converges, it converges to a steady state in the same regions as the initial price path of $\left(m_{0}, m_{0}\right)$. These states are $\left(m_{0}, p_{0}^{* *}\right)$ for $m_{o} \in R_{1},\left(m_{0}, m_{0}\right)$ for $m_{o} \in R_{2}$, and $(S, S)$ for $m_{o} \in R_{3}$.

Similarly, for scheme Pre, these steady states are $\left(m_{0}, p_{\text {pre }}^{* *}\right)$ for $m_{o} \in R_{\text {pre. } 1},\left(m_{0}, m_{0}\right) m_{o} \in R_{\text {pre. } 2}$ and $(S, S)$ for $m_{o} \in R_{\text {pre. } 3}$; for scheme Post, these steady states are $\left(m_{0}, p_{\text {post }}^{* *}\right)$ for $m_{o} \in$ $R_{\text {post.1 }},\left(m_{0}, m_{0}\right) m_{o} \in R_{\text {post. } 2}$ and $(S, S)$ for $m_{o} \in R_{\text {post. } 3 \text {. }}$.

We now turn to characterize the optimal price paths of problem (2), namely, under scheme $O$ which can also be applied to scheme Pre and scheme Post. For $m_{0} \in R_{1} \cup R_{2}$, $m_{t}=m_{0}$ by Proposition 10, so (2) can be rewritten (with $m_{o}$ as a parameter) as follows:

$$
V_{m_{0}}\left(p_{t-1}\right)=\max _{p_{t-1>m_{0}}}\left\{\prod\left(p_{t}, r_{t}\right)+\beta V_{m_{0}}\left(p_{t}\right)\right\}
$$

where $r_{t}=\eta m_{0}+(1-\eta) p_{t-1}$. That is, $V(m, p)=V_{m}(p)$ for $m_{0} \in R_{1} \cup R_{2}$ and $m<p$. Because $\prod\left(p_{t}, r_{t}\right)$ is supermodular, the optimal policy in $(2)$ is monotone, so $p_{t}^{*}\left(m_{0}, p_{t-1}\right)$ is increasing in $p_{t-1}$. There, the optimal path is monotonic in a bounded interval and hence converges to a steady state $\left(m_{0}, p^{* *}\left(m_{0}\right)\right)$.

For $m_{o} \in R_{3}$, we can get from Proposition 3 of [23] that the optimal price path is decreasing to $S$, by supermodularity of $\prod\left(p_{t}, r_{t}\right)$. Based on the analysis above, we can get the following conclusions.

Given initial state $\left(m_{0}, p_{0}\right)$ and scheme $O$, the optimal price path of (2) converges monotonically to a steady state, which is $\left(a_{1}\right) p^{* *}\left(m_{0}\right)$ if $m_{o} \in R_{1} ;\left(b_{1}\right) m_{o}$, if $m_{o} \in R_{2} ;\left(c_{1}\right)$ $S$, if $m_{0} \in R_{3}$. Under scheme Pre, the optimal price path of (5) converges monotonically to a steady state, which is $\left(a_{2}\right)$ $p_{\text {pre }}^{* *}\left(m_{0}\right)$, if $m_{o} \in R_{\text {pre. } 1} ;\left(b_{2}\right) m_{o}$, if $m_{o} \in R_{\text {pre. } 2} ;\left(c_{2}\right) S_{\text {pre }}$, if $m_{o} \in R_{\text {pre.3. }}$. Under scheme Post, the optimal price path of (7) converges monotonically to a steady state, which is $\left(a_{3}\right)$ $p_{\text {post }}^{* *}\left(m_{0}\right)$, if $m_{o} \in R_{\text {post. } 1} ;\left(b_{3}\right) m_{o}$, if $m_{o} \in R_{\text {post. } 2} ;\left(c_{3}\right) S_{\text {post }}$, if $m_{o} \in R_{\text {post. } 3 \text {. }}$

\section{Numerical Examples}

Based on Section 4, we know that in order to get the steady states under three schemes, respectively, we should know the $p^{* *}\left(m_{0}\right), s S$ under scheme $O, p_{\text {pre }}^{* *}\left(m_{0}\right), s_{\text {pre }} S_{\text {pre }}$ under scheme Pre, and $p_{\text {post }}^{* *}\left(m_{0}\right), s_{\text {post }} S_{\text {post }}$ under scheme Post.
According to (21), (22), and (24), we have

$$
\begin{aligned}
p^{* *}\left(m_{0}\right) & =\frac{\beta_{0}+\beta_{2} \eta m_{0}}{2 \beta_{1}+\beta_{2}(2-(1-\eta)(1+\beta))}, \\
s & =\frac{\beta_{0}}{2 \beta_{1}+\beta_{2}(1-\beta(1-\eta))}, \\
S & =\frac{\beta_{0}}{2 \beta_{1}+\beta_{2}(1-\beta)} .
\end{aligned}
$$

Based on Proposition 8 and (28), we have

$$
\begin{aligned}
p_{\text {pre }}^{*}\left(m_{0}\right) & =\frac{\beta_{0}+\beta_{2} \eta m_{0}}{2(1-\gamma) \beta_{1}+\beta_{2}(1-\gamma)(2-(1-\eta)(1+\beta))}, \\
s_{\text {pre }} & =\frac{\beta_{0}}{2(1-\gamma) \beta_{1}+\beta_{2}(1-\gamma)(1-\beta(1-\eta))}, \\
S_{\text {pre }} & =\frac{\beta_{0}}{2(1-\gamma) \beta_{1}+\beta_{2}(1-\gamma)(1-\beta)} .
\end{aligned}
$$

Based on Proposition 9 and (32), we have

$$
\begin{aligned}
p_{\text {Post }}^{*}\left(m_{0}\right) & =\frac{\beta_{0}+\beta_{2} \eta m}{2 \delta \beta_{1}+\beta_{2} \delta(1-\gamma)(1-\beta)}, \\
s_{\text {post }} & =\frac{\beta_{0}}{2 \delta \beta_{1}+\beta_{2} \delta(1-\gamma)(1-\beta)}, \\
S_{\text {post }} & =\frac{\beta_{0}}{2 \delta \beta_{1}+\beta_{2} \delta(1-\beta)} .
\end{aligned}
$$

In order to analyze how the steady states under three schemes change with the parameters, we set $\beta=0.95$, $\beta_{0}=20, \beta_{1}=20, \beta_{2}=40, \gamma=0.3, \varphi=0.6, \eta=0.5$, and $p=[p, \bar{p}]=[0.2,1]$. Then we can get Figures $1-3$.

From Figure 1, we know that under scheme $O, s=0.33$, $S=0.48$; namely, $R_{1}=[0.2,0.33], R_{2}=[0.33,0.48]$, and $R_{3}=[0.48,1]$ which means if $m_{0} \geq 0.48$, the steady price will be 0.48 ; if $0.33<m_{0}<0.48$, the steady price will be $m_{o}$; and if $m_{0}<0.33, p^{* *}\left(m_{0}\right)$ will be the steady state. From Figure 2, we can see under scheme Pre $s_{\text {pre }}=0.47$, $S_{\text {pre }}=0.68$, which means if $m_{o} \geq 0.68$, the steady price will be 0.68 ; if $0.47 \leq m_{o}<0.68$, the steady price will be $m_{o}$; and if $m_{o}<0.47, p^{* *}\left(m_{0}\right)$ will be the steady state. Figure 3 shows that if $0.55 \leq m_{o}<0.79$, the steady price will be $m_{o}<0.55$, and $p^{* *}\left(m_{0}\right)$ will be the steady state.

With further analysis, we can find out that $s_{\text {pre }}=s /(1-\gamma)$ and $s_{\text {post }}=s / \varphi$ and $S_{\text {pre }}=s /(1-\gamma)$ and $S_{\text {post }}=S / \varphi$ which can also be obtained in two-period dynamic pricing model. And under scheme $O$, scheme Pre, and scheme Post, the steady price converges monotonically. Besides, the characteristics of the convergence depend on $m_{0}$.

Next, we will investigate how the monopolist's steady state under scheme Pre varies with the pain buffering coefficient $\gamma$ and moreover how the steady state under scheme Post varies with the consumers' discount factor $\varphi$. Firstly, we should set the value of $m_{0}$. For the characteristics of convergence, we 


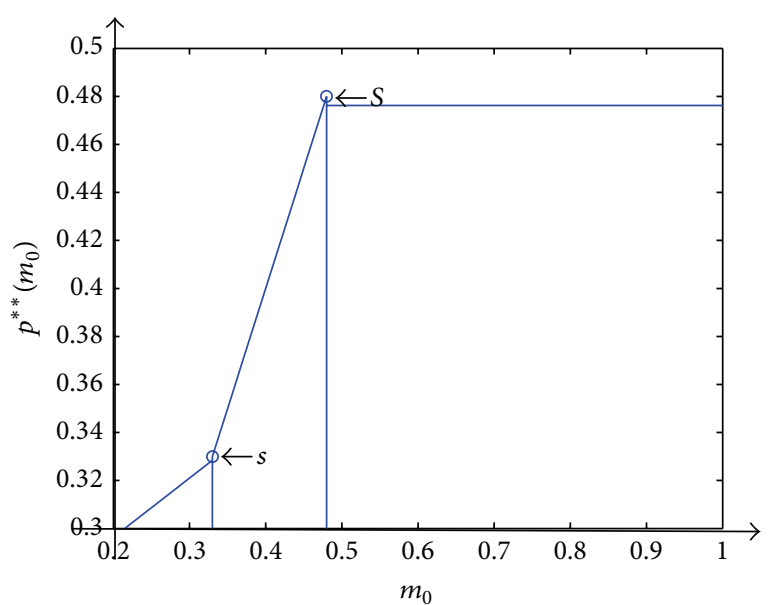

FIGURE 1: The steady price under scheme $O$.

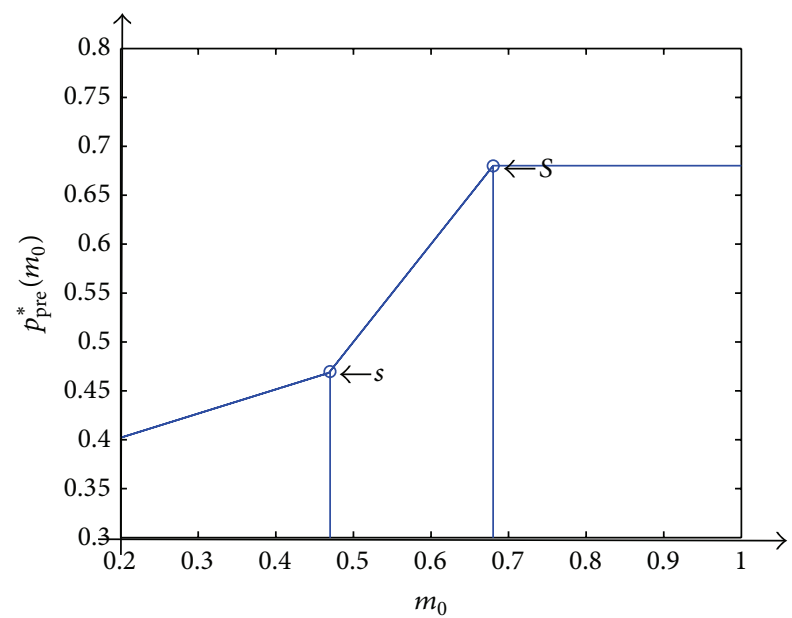

FIgUre 2: The steady state under scheme Pre.

just consider the case when the value of $m_{0}$ is small, such as 0.3 . Then we can get Figures 4 and 5 .

From Figure 4, we know that the bigger pain buffering coefficient $\gamma$ is, the higher the steady price under the scheme Pre is, which means that if the pain felt by consumers is much, the firm will be better to set the price high when they choose the scheme Pre. From Figure 5 we see that the less discount factor $\varphi$ is, the higher the steady price under the scheme Post is, which means that if consumers perceive a little discount, the firm will be better to set the price high when they choose the scheme Post.

\section{Conclusion}

This paper provides a new product demand forecasting and pricing model for consumers with double-entry mental accounting and peak-end anchoring model. With the assumption of linear demand, the model derives the steady price for three different payment schemes for two- and infinite-period, respectively. In infinite-period model, the characteristic of the steady state under different scheme has

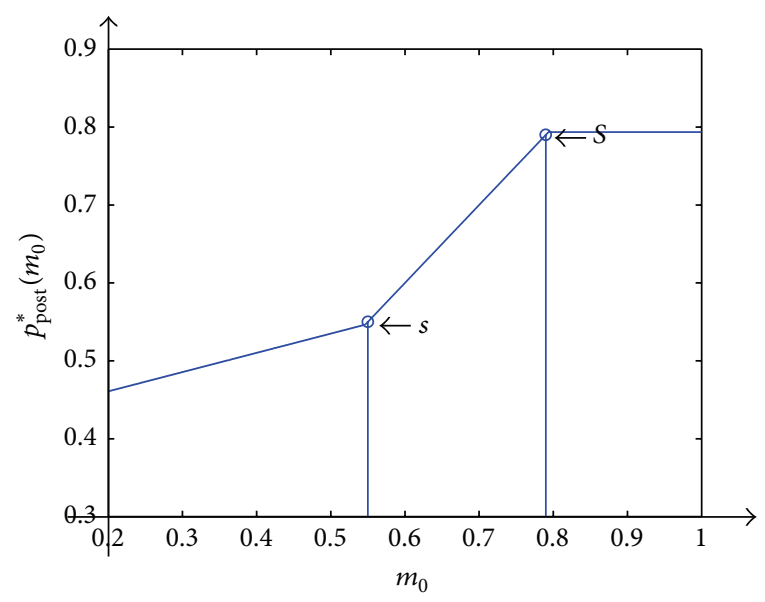

Figure 3: The steady state under scheme Post.

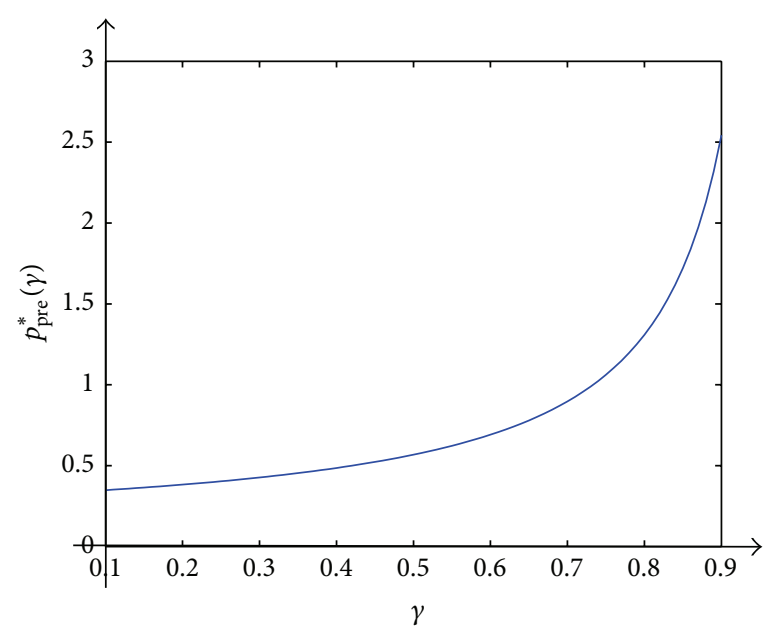

Figure 4: The steady state changing with $\gamma$ under scheme Pre.

something to do with $m_{0}$; the firm should set different scheme based on $m_{0}$ to make more profit. Besides, how the long-term profits of different schemes change with double-entry mental accounting's parameters and the advice about how to choose the best payment scheme for higher profits are provided via numerical analysis.

We demonstrate that consumers' mental accounting has significant impacts on product demand. It is necessary for a strategic company to consider it to get an accurate prediction of product demand. Future research can explore modeling consumers' mental accounting in other ways; for example, explore how reference price adaption is influenced by mental accounting [7].

\section{Appendices}

\section{A. The Proof of Proposition 4}

Case 1. Because $m_{0}=\max \left(m_{0}, p_{1},(1-\gamma) p_{1}, \delta p_{1}\right)$, in the scheme $O$, we have $m_{1}=\min \left(m_{0}, p_{1}\right)=p_{1}, r_{2}=\eta m_{1}+$ $(1-\eta) p_{1}=p_{1}$. 

tion.

Then, we can solve model (12) through backward induc-

Firstly, let $\partial \Pi_{o}\left(p_{2}, r_{2}\right) / \partial p_{2}=0$ and we can get the second period's optimal price formation: $p_{0.2}^{*}=\left(\beta_{0}+\beta_{2} p_{1}\right) / 2\left(\beta_{1}+\right.$ $\left.\beta_{2}\right)$.

Secondly, substituting $p_{0.2}^{*}$ into model (12) and letting $\partial V_{0}\left(r_{1}\right) / \partial p_{1}=0$ give the first period's specific optimal price $p_{0.1}^{*}=\left(2\left(\beta_{0}+\beta_{2} m_{0}\right)\left(\beta_{1}+\beta_{2}\right)+\beta \beta_{0} \beta_{2}\right) /\left(4\left(\beta_{2}+\beta_{2}\right)^{2}-\beta \beta_{2}^{2}\right)$.

Thirdly, substitute the specific $p_{o, 1}^{*}$ into formation $p_{o, 2}^{*}$, and we can obtain the second period's specific optimal price $p_{0.2}^{*}=\left(\beta_{0}+\beta_{2} p_{0.1}^{*}\right) / 2\left(\beta_{1}+\beta_{2}\right)$.

In the scheme Pre, we also have $m_{1}=\min \left(m_{0}, p_{1}\right)=p_{1}$; then $r_{2}=\eta m_{1}+(1-\eta)(1-\gamma) p_{1}=(1-\gamma) p_{1}$. Using the same ways above, we have $p_{\text {pre. }}^{*}=\left(2\left(\beta_{0}+\beta_{2} m_{0}\right)\left(\beta_{1}+\beta_{2}\right)+\right.$ $\left.\beta \beta_{0} \beta_{2}\right) /\left(4(1-\gamma)\left(\beta_{2}+\beta_{2}\right)^{2}-(1-\gamma) \beta \beta_{2}^{2}\right), p_{\text {pre. } 2}^{*}=\left(\beta_{0}+\beta_{2}(1-\right.$ $\left.\gamma) p_{1}\right) /\left(2(1-\gamma)\left(\beta_{1}+\beta_{2}\right)\right)$.

In the scheme Post, we have $r_{2}=\eta m_{1}+(1-\eta) \delta p_{1}=$ $\delta p_{1}$; similarly, we have $p_{\text {post.1 }}^{*}=\left(2\left(\beta_{0}+\beta_{2} m_{0}\right)\left(\beta_{1}+\beta_{2}\right)+\right.$ $\left.\beta \beta_{0} \beta_{2}\right) /\left(4 \delta\left(\beta_{2}+\beta_{2}\right)^{2}-\delta \beta \beta_{2}^{2}\right), p_{\text {post. } 2}^{*}=\left(\beta_{0}+\beta_{2} \delta p_{1}\right) / 2 \delta\left(\beta_{1}+\right.$ $\left.\beta_{2}\right)$. So we have $p_{\text {pre.1 }}^{*}=p_{0.1}^{*} /(1-\gamma), p_{\text {pre. } 2}^{*}=p_{0.2}^{*} /(1-\gamma)$, $p_{\text {post. } 1}^{*}=p_{0.1}^{*} / \delta$, and $p_{\text {post. } 2}^{*}=p_{0.2}^{*} / \delta$.

Case 2. Because of $m_{0}=\min \left(m_{0}, p_{1},(1-\gamma) p_{1}, \delta p_{1}\right)$ in the scheme $O$, we have $m_{1}=\min \left(m_{0}, p_{1}\right)=m_{0}$; then $r_{2}=\eta m_{1}+$ $(1-\eta) p_{1}=\eta m_{0}+(1-\eta) p_{1}$.

Using the same ways above, we can get

$$
\begin{aligned}
& p_{0.1}^{*}=\frac{2\left(\beta_{0}+\beta_{2} m_{0}\right)\left(\beta_{1}+\beta_{2}\right)+\beta\left(\beta_{0}+\beta_{2} \eta m_{0}\right) \beta_{2}(1-\eta)}{4\left(\beta_{2}+\beta_{2}\right)^{2}-\beta \beta_{2}^{2}(1-\eta)^{2}}, \\
& p_{0.2}^{*}=\frac{\beta_{0}+\beta_{2}\left[\eta m_{0}+(1-\eta) p_{1}\right]}{2\left(\beta_{1}+\beta_{2}\right)}, \\
& p_{\text {pre. }}^{*}=\frac{2\left(\beta_{0}+\beta_{2} m_{0}\right)\left(\beta_{1}+\beta_{2}\right)+\beta\left(\beta_{0}+\beta_{2} \eta m_{0}\right) \beta_{2}(1-\eta)}{4\left(\beta_{2}+\beta_{2}\right)^{2}(1-\gamma)-\beta \beta_{2}^{2}(1-\eta)^{2}(1-\gamma)}, \\
& p_{\text {pre.2 }}^{*}=\frac{\beta_{0}+\beta_{2}\left[\eta m_{0}+(1-\eta)(1-\gamma) p_{1}\right]}{2(1-\gamma)\left(\beta_{1}+\beta_{2}\right)} \\
& p_{\text {post.1 }}^{*} \\
& =\frac{2\left(\beta_{0}+\beta_{2} m_{0}\right)\left(\beta_{1}+\beta_{2}\right)+\beta\left(\beta_{0}+\beta_{2} \eta m_{0}\right) \beta_{2}(1-\eta) \delta}{4\left(\beta_{2}+\beta_{2}\right)^{2} \delta-\beta \beta_{2}^{2}(1-\eta)^{2} \delta}, \\
& p_{\text {post.2 }}^{*}=\frac{\beta_{0}+\beta_{2}\left[\eta m_{0}+(1-\eta) \delta p_{1}\right]}{2 \delta\left(\beta_{1}+\beta_{2}\right)} .
\end{aligned}
$$

So we also can get $p_{\text {pre.1 }}^{*}=p_{0.1}^{*} /(1-\gamma), p_{\text {pre. }}^{*}=p_{0.2}^{*} /(1-\gamma)$, $p_{\text {post. } 1}^{*}=p_{0.1}^{*} / \delta$, and $p_{\text {post. } 1}^{*}=p_{0.1}^{*} / \delta$.

\section{B. The Proof of Proposition 5}

In Case 1 of Proposition 4, namely, $m_{0}=\max \left(m_{0}, p_{1},(1-\right.$ $\left.\gamma) p, \delta p_{1}\right)$, we have $r_{2}=p_{1}$. According to $p_{o, 1}^{*}, p_{o, 2}^{*}$, the

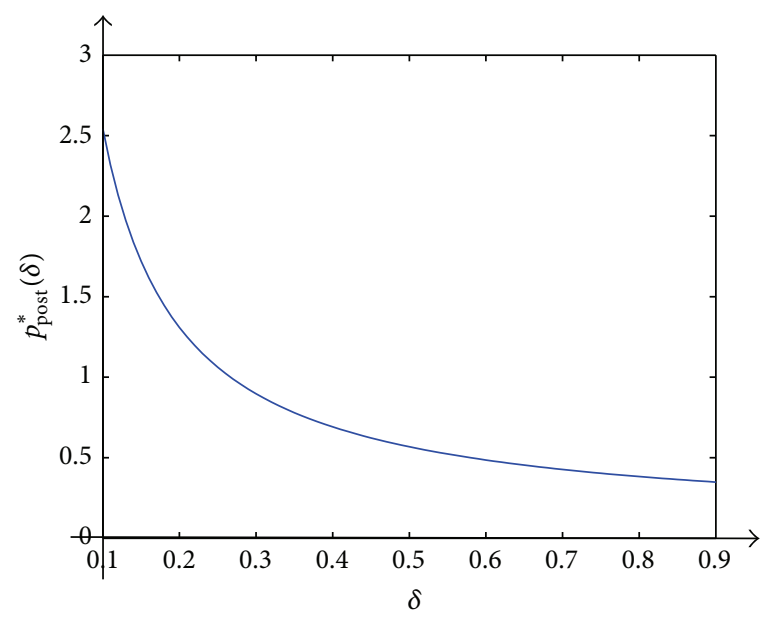

Figure 5: The steady state changing with $\varphi$ under scheme Post.

maximum profit of model (12) is $V_{0}^{*}\left(m_{0}\right)=\prod_{0}\left(p_{0.1}^{*}, r_{1}^{*}\right)+$ $\beta \prod_{0}\left(p_{0.2}^{*}, r_{2}^{*}\right)$, where $r_{1}=m_{0}$ and $r_{2}^{*}=p_{0.1}^{*}$.

According to model (13), we have

$$
\begin{aligned}
V_{\text {pre }}^{*}\left(m_{0}\right)=p_{\text {pre. } 1}^{*}[ & \beta_{0}-(1-\gamma) p_{\text {pre. } 1}^{*} \\
+ & \left.\beta_{2}\left(r_{1}-(1-\gamma) p_{\text {pre. } 1}^{*}\right)\right] \\
+\beta p_{\text {pre.2 }}^{*} & {\left[\beta_{0}-\beta_{1}(1-\gamma) p_{\text {pre. } 2}^{*}\right.} \\
& \left.+\beta_{2}\left(r_{2}-(1-\gamma) p_{\text {pre. } 2}^{*}\right)\right] .
\end{aligned}
$$

Substituting $p_{\text {pre. } 1}^{*}=p_{0.1}^{*} /(1-\gamma), p_{\text {pre. } 2}^{*}=p_{0.2}^{*} /(1-\gamma)$ into $V_{\text {pre }}^{*}\left(m_{0}\right)$, we obtain

$$
\begin{aligned}
V_{\mathrm{pre}}^{*}\left(m_{0}\right)= & \frac{p_{0.1}^{*}}{1-\gamma}\left[\beta_{0}-\beta_{1} p_{0.1}^{*}+\beta_{2}\left(r_{1}-(1-\gamma) p_{0.1}^{*}\right)\right] \\
& +\beta \frac{p_{0.2}^{*}}{1-\gamma}\left[\beta_{0}-\beta_{1} p_{0.2}^{*}+\beta_{2}\left(p_{0.1}^{*}-p_{0.2}^{*}\right)\right] \\
= & \frac{V_{0}^{*}\left(m_{0}\right)}{1-\gamma} .
\end{aligned}
$$

Similarly, we can get $V_{\text {post }}^{*}\left(m_{0}\right)=V_{0}^{*}\left(m_{0}\right) / \varphi$.

\section{Proof Proposition 7}

We first show that $p^{* *}(m)$, as defined by (24), is feasible; that is, $p^{* *}(m) \geq m$ for $m \in[p, s]$. Note that $p^{* *}(m)$ is increasing in $m$ and single-crosses the single identity line from above at $s$, defined by (21). Feasibility follows because (24) has a unique positive solution $p^{* *}(p)$ at $m=0$.

For $m \in[p, s]$, the constant pricing policy $p \equiv p^{* *}(m)$ is optimal for problem (20) with $w=0$ and feasible for (2). Because $m \leq s, \min \left(m, p^{* *}(m)\right)=m$ and $r=\eta m+$ 
$(1-\eta) p^{* *}(m) \leq p^{* *}(m)$, which implies $\prod\left(p_{t}, r_{t}\right)=(1-$ $\omega) \prod\left(p_{t}, \eta m+(1-\eta) p_{t-1}\right)+\omega \prod\left(p_{t}, p_{t-1}\right)=\prod\left(p_{t}, \eta m+(1-\right.$ $\left.\eta) p_{t-1}\right)$. This constant pricing policy yields the same value in both problems, so it is also optimal for $(2)$, and $\left(m, p^{* *}(m)\right)$ is a steady state of (2).

For $\in[s, S]$, the constant pricing policy $p_{t} \equiv m$ is optimal for (20), is feasible for (2), and yields the same value in both problems. Therefore $(m, m)$ is the steady state of $(2)$.

\section{Conflict of Interests}

The authors declare that there is no conflict of interests regarding the publication of this paper.

\section{Acknowledgment}

This research is supported by National Natural Science Foundation of China (Grant nos. 71371191, 71221061, and 71210003).

\section{References}

[1] N. Mahbub, S. K. Paul, and A. Azeem, "A neural approach to product demand forecasting," International Journal of Industrial and Systems Engineering, vol. 15, no. 1, pp. 1-18, 2013.

[2] L. Wang, C. X. Dun, W. J. Bi, and Y. R. Zeng, "An effective and efficient differential evolution algorithm for the integrated stochastic joint replenishment and delivery model," KnowledgeBased Systems, vol. 36, pp. 104-114, 2012.

[3] J. Huang, M. Leng, and M. Parlar, "Demand functions in decision modeling: a comprehensive survey and research directions," Decision Sciences, vol. 44, no. 3, pp. 557-609, 2013.

[4] W. Bi, L. Tian, H. Liu, and X. Chen, "A stochastic dynamic programming approach based on bounded rationality and application to dynamic portfolio choice," Discrete Dynamics in Nature and Society, vol. 2014, Article ID 840725, 11 pages, 2014.

[5] W. Bi, Y. Sun, H. Liu, and X. Chen, "Dynamic nonlinear pricing model based on adaptive and sophisticated learning," Mathematical Problems in Engineering, vol. 2014, Article ID 791656, 11 pages, 2014.

[6] R. H. Thaler, "Mental accounting matters," Journal of Behavioral Decision Making, vol. 12, no. 3, pp. 183-206, 1999.

[7] M. Baucells and W. Hwang, "A Model of Mental Accounting and Reference Price Adaptation," 2013, http://ssrn.com/abstract= 2283884.

[8] R. H. Thaler, "Mental accounting and consumer choice," Marketing Science, vol. 4, no. 3, pp. 199-214, 1985.

[9] D. Prelec and G. Loewenstein, "The red and the black: mental accounting of savings and debt," Marketing Science, vol. 17, no. 1, pp. 4-28, 1998.

[10] N. Barberis and M. Huang, "Mental accounting, loss aversion, and individual stock returns," The Journal of Finance, vol. 56, no. 4, pp. 1247-1292, 2001.

[11] T. Langer and M. Weber, "Prospect theory, mental accounting, and differences in aggregated and segregated evaluation of lottery portfolios," Management Science, vol. 47, no. 5, pp. 716733, 2001.

[12] S. Frederick, G. Loewenstein, and T. O’Donoghue, “Time discounting and time preference: a critical review," Journal of Economic Literature, vol. 40, no. 2, pp. 351-401, 2002.
[13] M. Grinblatt and B. Han, "Prospect theory, mental accounting, and momentum," Journal of Financial Economics, vol. 78, no. 2, pp. 311-339, 2005.

[14] Y. Liu, C. Ding, C. Fan, and X. Chen, "Pricing decision under DUAl-channel structure considering fairness and FREe-riding behavior," Discrete Dynamics in Nature and Society, vol. 2014, Article ID 536576, 10 pages, 2014.

[15] E. Shafir and R. H. Thaler, "Invest now, drink later, spend never: on the mental accounting of delayed consumption," Journal of Economic Psychology, vol. 27, no. 5, pp. 694-712, 2006.

[16] U. Simonsohn and F. Gino, "Daily horizons: evidence of narrow bracketing in judgment from 10 years of MBA admissions interviews," Psychological Science, vol. 24, no. 2, pp. 219-224, 2013.

[17] S. Erat and S. R. Bhaskaran, "Consumer mental accounts and implications to selling base products and add-ons," Marketing Science, vol. 31, no. 5, pp. 801-818, 2012.

[18] L. Chen, A. G. Kök, and J. D. Tong, "The effect of payment schemes on inventory decisions: the role of mental accounting," Management Science, vol. 59, no. 2, pp. 436-451, 2013.

[19] M. Liu, W. Bi, X. Chen, and G. Li, "Dynamic pricing of fashionlike multiproducts with customers' reference effect and limited memory," Mathematical Problems in Engineering, vol. 2014, Article ID 157865, 10 pages, 2014.

[20] I. Popescu and Y. Wu, "Dynamic pricing strategies with reference effects," Operations Research, vol. 55, no. 3, pp. 413-429, 2007.

[21] R. S. Winer, "A reference price model of brand choice for frequently purchased products," Journal of Consumer Research, vol. 13, no. 2, pp. 250-256, 1986.

[22] E. A. Greenleaf, "The impact of reference price effects on the profitability of price promotions," Marketing Science, vol. 14, no. 1, pp. 82-104, 1995.

[23] J. Nasiry and I. Popescu, "Dynamic pricing with loss-averse consumers and peak-end anchoring," Operations Research, vol. 59, no. 6, pp. 1361-1368, 2011. 


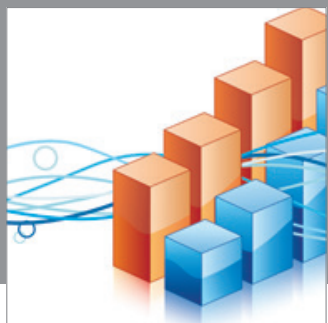

Advances in

Operations Research

mansans

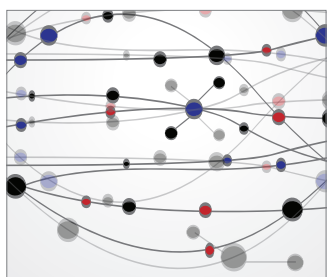

The Scientific World Journal
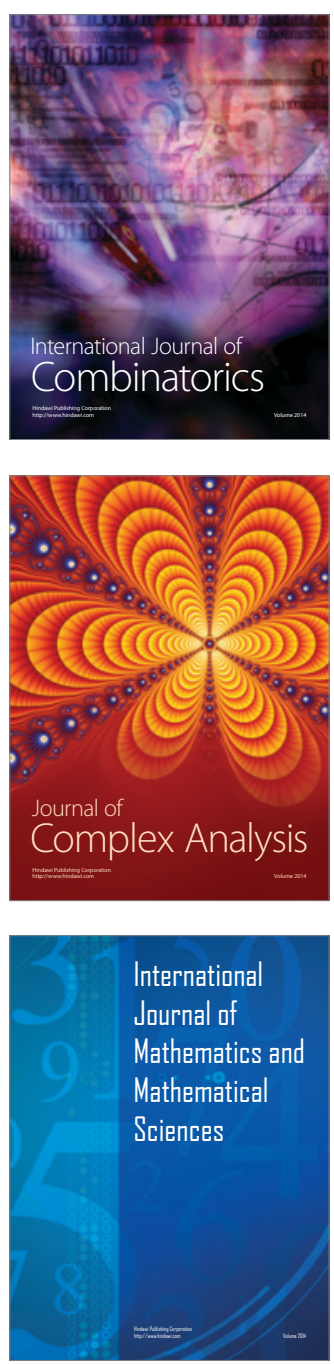
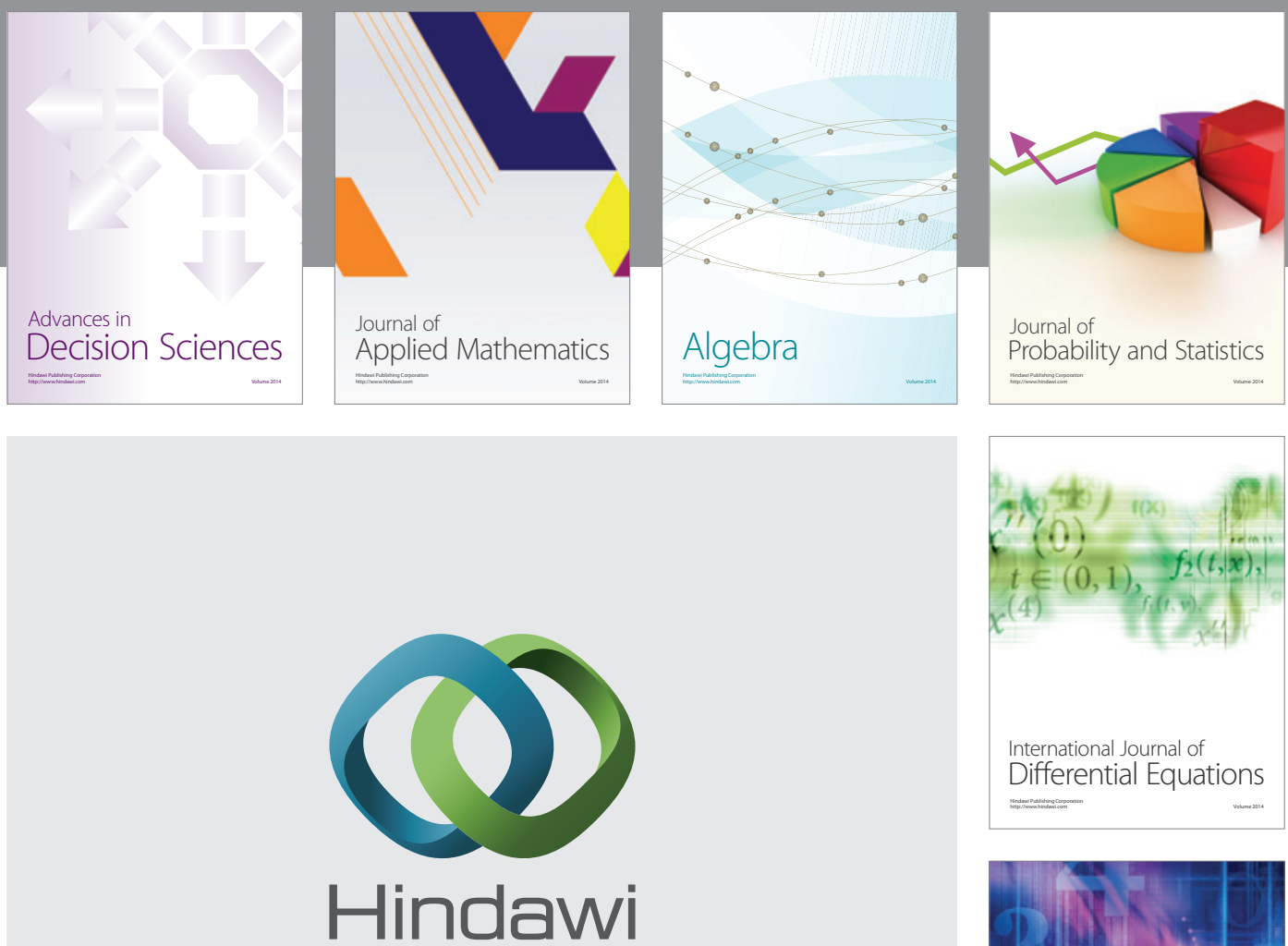

Submit your manuscripts at http://www.hindawi.com
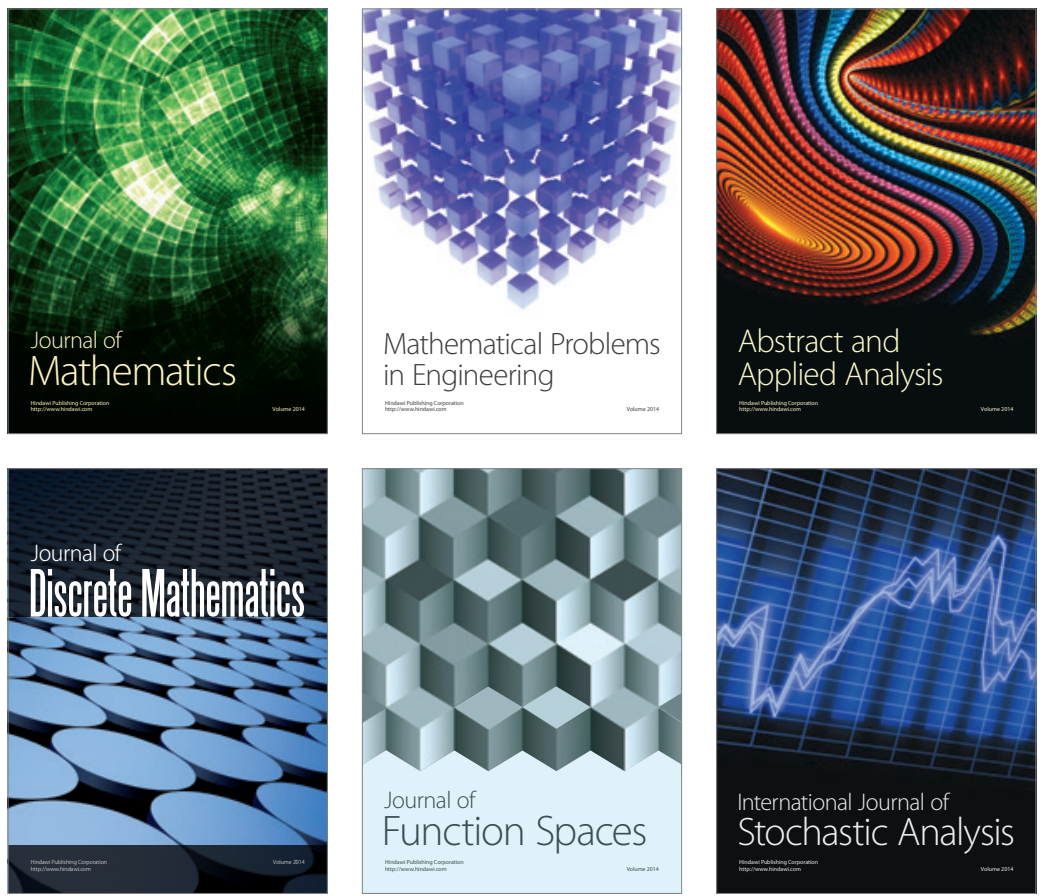

Journal of

Function Spaces

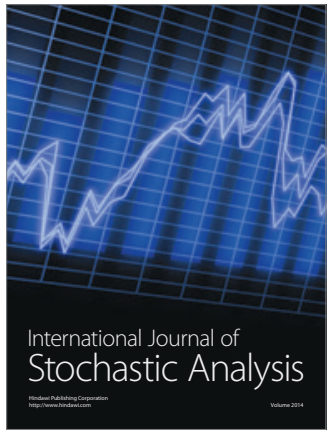

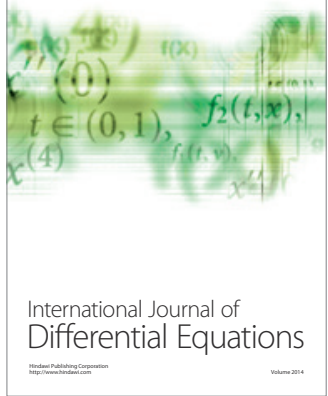
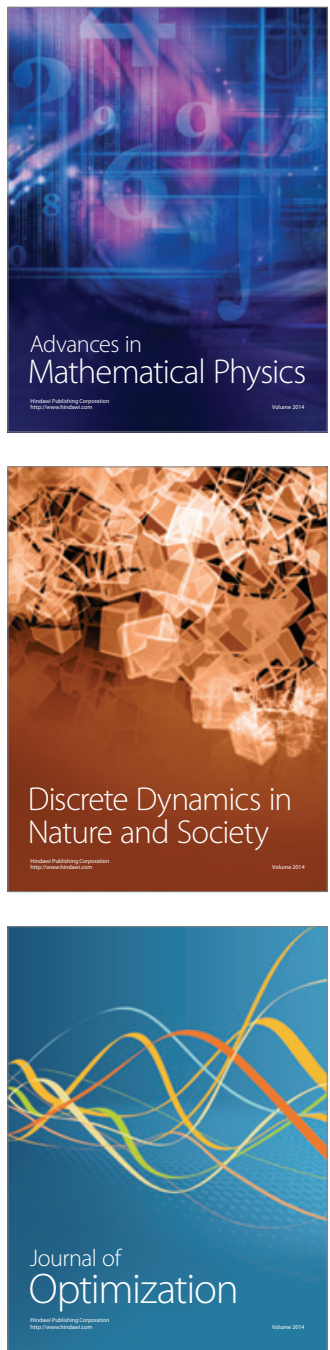